\title{
Development of Wavelength Shifters for the ArDM Argon Dark Matter Detector
}

\author{
Konstantinos Mavrokoridis ${ }^{* \dagger}$ \\ Department of Physics and Astronomy, University of Sheffield, \\ Hicks Building, Hounsfield Road, Sheffield, S3 7RH, United Kingdom \\ E-mail: k.mavrokoridis@shef.ac.uk
}

\begin{abstract}
Wavelength shifting organic fluors such as tetraphenyl butadiene (TPB) can shift VUV and UV light into the visible blue high quantum efficiency region of low cost borosilicate windowed bialkali photomultiplier tubes (PMTs). Various thicknesses of TPB were deposited by spraying and vacuum evaporation onto both specular $3 \mathrm{M}^{\mathrm{TM}}$-foil and diffuse Tetratex ${ }^{\mathrm{TM}}$ (TTX) reflectors. $128 \mathrm{~nm}$ VUV light generated in 1 bar argon gas by a $5.4 \mathrm{MeV} \alpha$ source was detected by a 3-inch bialkali borosilicate PMT within $1 \mathrm{~m}$ tube lined internally with a TPB coated reflector. The light collection was recorded as a function of separation between source and PMT for each combination of coating and reflector for distances up to $1 \mathrm{~m}$. Finally the PMT window was coated in order to shift direct VUV light and the relative efficiencies of each application were compared. The optimum coating and reflector combination was TPB evaporated on TTX. Measurements with coating thicknesses of $0.2 \mathrm{mg} / \mathrm{cm}^{2}$ and $1.0 \mathrm{mg} / \mathrm{cm}^{2}$ yielded similar performance. The best PMT window coating is obtained by TPB evaporation of $0.05 \mathrm{mg} / \mathrm{cm}^{2}$.
\end{abstract}

Identification of dark matter 2008

August 18-22, 2008

Stockholm, Sweden

\footnotetext{
*Speaker.

On behalf of ArDM collaboration.
} 


\section{Introduction}

Tonne scale liquid argon (LAr) targets such as that used in the Argon Dark Matter (ArDM) experiment $[1,2]$ typically require in excess of ten large area PMTs for acceptable light readout. The argon scintillation light due to neutral or charged particle excitation is in the vacuum ultraviolet (VUV) centered at about $128 \mathrm{~nm}[3,4,5]$. Currently large $\mathrm{MgF}_{2}$ windowed PMTs that can detect $128 \mathrm{~nm}$ light are commercially not available. A common alternative technique is to apply wavelengh shifting chemicals on borosilicate windowed PMTs thus shifting VUV argon scintillation into the visible spectrum, the typical quantum efficiency (QE) of a borosilicate windowed PMT being from 10 to $15 \%$ at approximately $430 \mathrm{~nm}$. Tetraphenyl butadiene (TPB) powder has been used which has an above average Stokes shift and can absorb $128 \mathrm{~nm}$ light, emitting in the required visible PMT region $[6,7,8,9]$. The argon scintillation light, is characterised by two distinct decay times - a slow component, $\tau_{2}$ (triplet eximer), and a fast component, $\tau_{1}$ (single eximer) $[4,10]$. Determination of the time constants is through a multiple parameters nonlinear least square fit with additional degrees of freedom related to the height, start time and baseline of the pulses. The decay time of the slow component, $\tau_{2}$, increases with the increase of argon purity and therefore can be used as a measure of the purity of argon. The observed effect of the purity on the slow component decay time has been hypothesized to be due to water impurities colliding with the long lived triple state [11]. From the literature the purest gas argon has a $\tau_{2}$ of $3200 \pm 300 \mathrm{~ns}$ [10]. The analysis of the measurements described in subsections 3.1, 3.2 was based around this property of argon scintillation. Section 2 describes TPB coating methods and sample preparations. Section 3 details two experiments aiming for reflector selection, optimisation of TPB coating thickness and deposition technique and presents the results obtained.

\section{WLS coating techniques and Reflectors}

\subsection{Reflector type}

Our research focussed on the two materials ESR (Vikuiti ${ }^{\mathrm{TM}}$ Enhanced Specular Reflector foil) from the company $3 \mathrm{M}$ and Tetratex ${ }^{\mathrm{TM}}$ (TTX) from the company Donaldson Membranes. $3 \mathrm{M}$ foil is a multilayer specular reflecting polymer film and as such is likely to be of high radio-purity. Its appearance is that of a polished metal although the material is non conducting by its nature. It has a specular reflection coefficient of approximately $100 \%$ in a large region of the optical spectrum. TTX is an aligned polytetrafluoroethylene (PTFE) fibrous cloth and is nearly a 100\% diffuse lambertian reflector. The TTX cloth used during these measurements was of $254 \mu \mathrm{m}$ thickness, similar types previously used to wrap $\mathrm{NaI}$ crystals.

\subsection{TPB deposition techniques on substrates and PMT windows}

TPB powder can be applied to a reflector or PMT window by vacuum evaporation, spraying, or by dissolving in a polymer matrix [6, 7]. Vacuum evaporation was performed in an Edwards model E308 evaporation chamber. TPB powder, which has a melting point of $207^{\circ} \mathrm{C}$, was heated electrically in the vacuum chamber by applying $24 \mathrm{~A}$ current to a molybdenum sample holder containing up to $3 g$ of powder. The reflector/PMT window was placed above the TPB powder at a fixed distance and the coating thickness was controlled by varying this distance and the weight 
of the powder. Sprayed coatings were prepared by dissolving TPB in toluene in a ratio of 1 to 40. This solution was then airbrushed onto the substrate using 1.2 bar argon gas. The polymer matrix coatings were prepared using long chain paraloid or polystyrene plastic fragments dissolved in toluene. An amount of TPB was added and dissolved isotropically. A known amount of liquid was then syringed onto the substrate. The TPB concentration within the solution was varied, as was the amount of liquid applied to the substrate. The solution was left for three hours to allow the toluene to evaporate, forming clear TPB impregnated plastic.

\section{Experimental procedures and results}

\subsection{Global efficiency of wavelength shifting and reflection with distance}

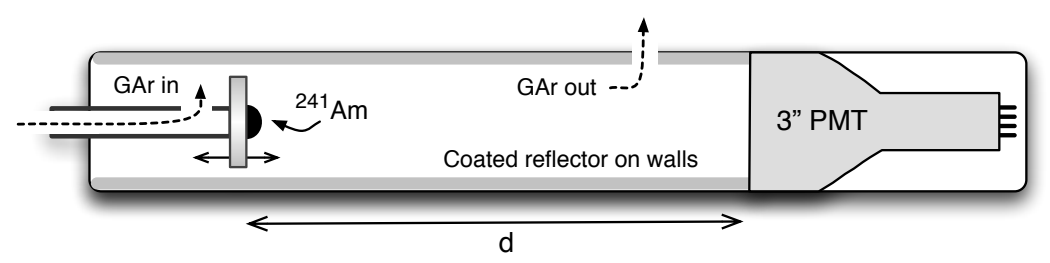

Figure 1: A schematic illustration of the argon gas apparatus used to determine the wavelength shifting and reflection efficiency with distance.

A schematic illustration of the argon gas apparatus which was used is shown in Figure 1. This apparatus consisted of a sealed polyvinyl chloride (PVC) tube containing a 3 inch uncoated PMT (electron tube type 9302KB). An $\alpha$ source located at the centre of a TPB coated reflector disk was placed within the tube at a fixed distance from the PMT (the total deposition of alpha energy occurs within $4 \mathrm{~cm}$ in 1 bar of gaseous argon). Samples of either 3M foil or TTX cloth coated with TPB were placed around the interior walls of the tube. A delivery tube was inserted into the PVC chamber and $99.9999 \%$ pure argon gas at 1 bar flowed throughout the apparatus. The argon flow rate was used to control the argon purity. Measurements were taken for varied TPB thicknesses between $0.2 \mathrm{mg} / \mathrm{cm}^{2}$ and $4.0 \mathrm{mg} / \mathrm{cm}^{2}$, which were deposited both via evaporation and spraying. Additionally, the distance between the $\alpha$ source and the PMT was altered in order to investigate the effect of both the attenuation of light following multiple reflections and the reduction in direct VUV light incident on the PMT. The number of photoelectrons collected at the PMT for each separation (defined as the total area of the light pulse) was then plotted against the slow component decay time $\left(\tau_{2}\right)$ for various distances $d$.

The results of the analysis are presented in Figure 2. The reduction in the total light collection with increasing distance was found, within errors, to be independent of TPB thickness and substrate. Evaporated coatings on $3 \mathrm{M}$ foil consistently underperformed irrespective of coating thickness compared to TTX cloth. Thicker coatings on 3M foil yielded higher light collection whereas light collection from TPB coated on TTX substrates was found to be almost independent of thickness. The $0.2 \mathrm{mg} / \mathrm{cm}^{2}$ TPB on TTX yielded within errors an identical result to the $1.0 \mathrm{mg} / \mathrm{cm}^{2}$ coating. 


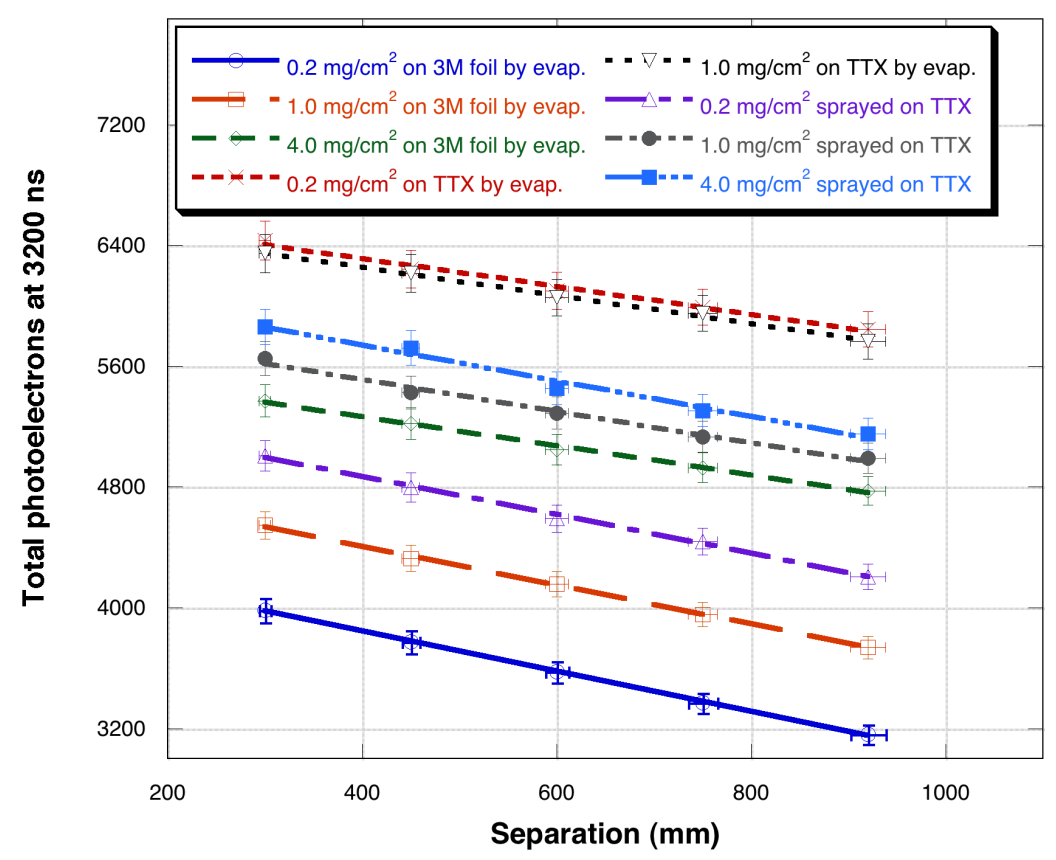

Figure 2: Total photoelectrons for 3200 ns purity against separation from the alpha source to PMT for TPB coated reflector walled tube.

\subsection{Wavelength shifting direct light incident on the PMT}

The argon gas apparatus for direct light measurements was constructed with a similar aspect ratio as the full scale ArDM target (Figure 3a). The experiment consisted of a sealed PVC tube containing a 3 inch coated PMT. The PMT window was coated with TPB powder with thicknesses ranging from $0.02 \mathrm{mg} / \mathrm{cm}^{2}$ to $2 \mathrm{mg} / \mathrm{cm}^{2}$ via evaporation, spraying and application of a polymer matrix containing TPB. The sides and base of the PVC tube were covered with $3 \mathrm{M}$ foil reflector coated with $1 \mathrm{mg} / \mathrm{cm}^{2}$ TPB powder by evaporation. TPB coated reflector walls were used as the ability of the window coating to shift VUV light is equally important as its ability to allow shifted visible light from the walls to penetrate. An $\alpha$ source was positioned $10 \mathrm{~cm}$ away from the PMT window and argon gas was flowed continually. The effect of various PMT window coatings on the total light collection was then recorded by plotting the slow component decay time $\left(\tau_{2}\right)$ against the total number of photoelectrons collected at the PMT.

Figure $3 \mathrm{~b}$ presents the results for the optimum PMT coatings. The optimum thickness was $0.05 \mathrm{mg} / \mathrm{cm}^{2}$ by evaporation, improving the total light collection by $28 \% \pm 0.8 \%$ at $1000 \mathrm{~ns} \mathrm{pu}-$ rity compared to that collected with no PMT coating. To avoid TPB crystallisation, deposition by spraying must be slow allowing evaporation of toluene. For polymer matrix coatings, crystallisation of TPB could be avoided with the addition of a plasticiser, which cross-links paraloid/polysterene chains with TPB, thus forming a rigid lattice while the solvent evaporates. Although the polystyrene matrices perform better than the paraloid, they may be unsuitable for use in ArDM target as PMTs are a source of radioactivity and polystyrene is a weak scintillator. 
a)

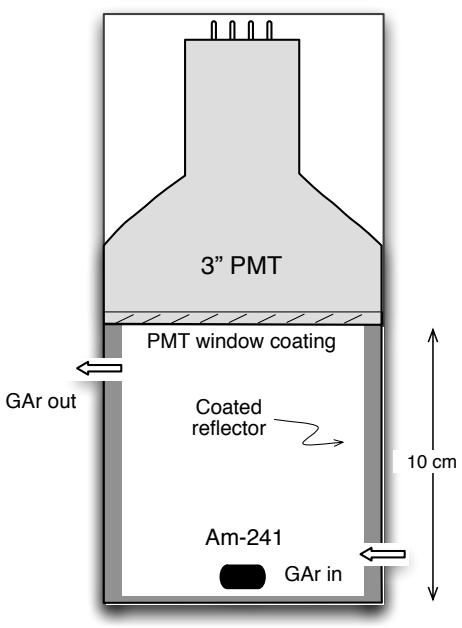

b)

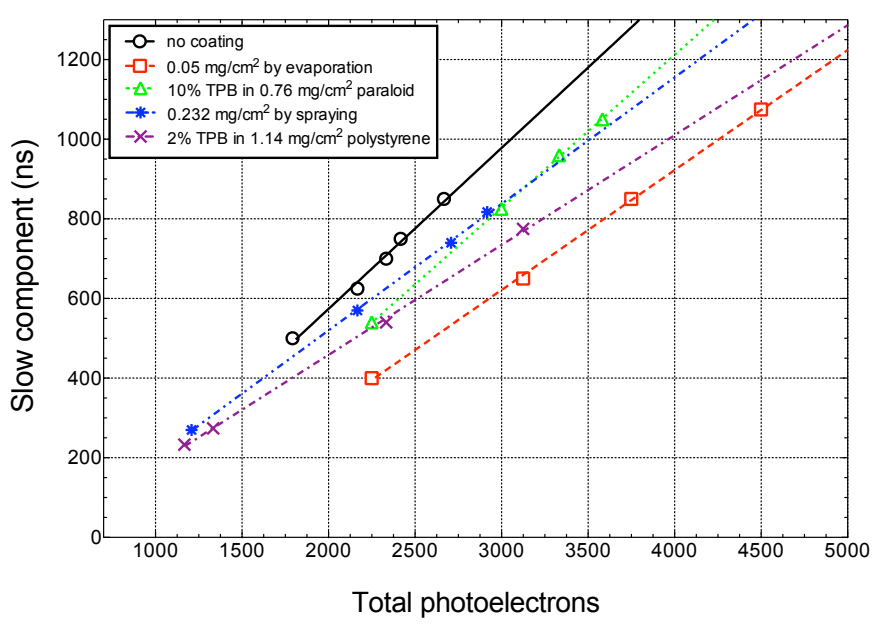

Figure 3: a) A schematic illustration of the argon gas apparatus used to determine the wavelength shifting efficiency of direct light incident on a TPB coated PMT. b) Results for the best PMT window coatings (polysterene and paraloid matrices, evaporation and spray).

\section{Conclusions}

TPB can efficiently absorb VUV radiation from argon luminescence and re-emit light in a wavelength which can be readily detected by bialkali photocathodes. TTX cloth was found to be a preferable reflector when compared with $3 \mathrm{M}^{\mathrm{TM}}$ foil due to its better light yield and large tolerance for the TPB layer thickness. Furthermore, the best TPB deposition method was found to be vacuum evaporation which avoids crystallisation and coating inhomogeneities. The best PMT window coating was found to be $0.05 \mathrm{mg} / \mathrm{cm}^{2} \mathrm{TPB}$ deposited via evaporation.

\section{References}

[1] M. Laffranchi and A. Rubbia. Journal of Physics: Conference Series, 65:2014, Apr 2007.

[2] A. Rubbia. J. Phys. Conf. Ser., 39:126, 2006.

[3] T. Doke et al. Nucl. Instr. and Meth., 291:617, Jun 1990.

[4] S. Kubota et al. Journal of Physics C, 11:2645, Jun 1978.

[5] S. Kubota et al. Physical Review B, 17:2762, Mar 1978.

[6] D. N McKinsey et al. Nucl. Instr. and Meth., 132:351, Nov 1997.

[7] G. J Davies et al. Nucl. Instr. and Meth., 117:421, Oct 1996.

[8] I.B. Berlman. Academic Press, New York and London, 1965.

[9] J. M Flournoy et al. Nucl. Instr. and Meth., 351:349, Dec 1994.

[10] J. W Keto et al. Phy. Rev. Let., 33:1365, Dec 1974.

[11] C. Amsler et al. JINST, 02:2001, Feb 2008. 